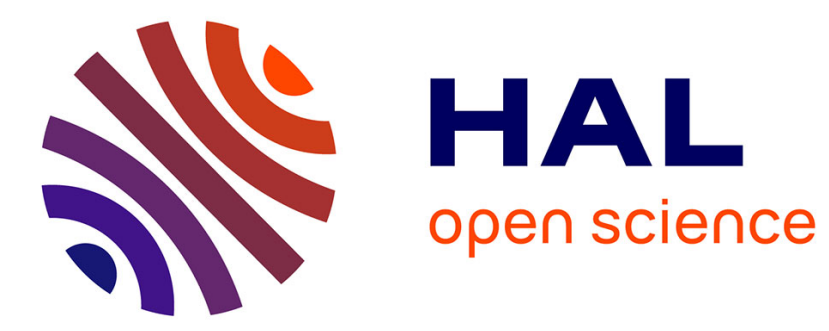

\title{
Weighted Consensus Protocols Design based on Network Centrality for Multi-agent Systems with Sampled-data
}

Myeongjin Park, Ohmin Kwon, Alexandre Seuret

\section{To cite this version:}

Myeongjin Park, Ohmin Kwon, Alexandre Seuret. Weighted Consensus Protocols Design based on Network Centrality for Multi-agent Systems with Sampled-data. IEEE Transactions on Automatic Control, 2017, 62 (6), pp.2916-2922. 10.1109/TAC.2016.2604682 . hal-01379401

\author{
HAL Id: hal-01379401 \\ https://hal.science/hal-01379401
}

Submitted on 12 Oct 2016

HAL is a multi-disciplinary open access archive for the deposit and dissemination of scientific research documents, whether they are published or not. The documents may come from teaching and research institutions in France or abroad, or from public or private research centers.
L'archive ouverte pluridisciplinaire HAL, est destinée au dépôt et à la diffusion de documents scientifiques de niveau recherche, publiés ou non, émanant des établissements d'enseignement et de recherche français ou étrangers, des laboratoires publics ou privés. 


\section{Weighted Consensus Protocols Design based on Network Centrality for Multi-agent Systems with Sampled-data}

\author{
MyeongJin Park, OhMin Kwon, Alexandre Seuret
}

\begin{abstract}
This paper aims at constructing and analyzing an efficient framework for the leader-following consensus protocol in multi-agent systems (MASs). We propose two novel consensus protocols weighted by calculating the betweenness and eigenvector centralities for agent and link which are determined by the interconnection structure of MASs. The concepts of centrality were introduced in the field of social science. Ultimately, the use of the proposed protocols can be described with regard to not only the number of each agent's neighbors, which was utilized in the existing works, but also more information about agents through considering two such centralities. By utilizing the Lyapunov method and some mathematical techniques, the leader-following guaranteed cost consensus conditions for MASs with the proposed protocols and sampleddata will be established in terms of linear matrix inequalities (LMIs). Based on the result of consensus criteria, two new protocol design methods which utilize the betweenness and eigenvector centralities will be proposed. Finally, some simulation results are given to illustrate the advantages of the proposed protocols in point of the robustness on sampling interval and the transient consensus performance.
\end{abstract}

Index Terms-Multi-agent Systems, Consensus, Betweenness and Eigenvector Centralities, Sampled-data, Lyapunov method, LMI.

\section{INTRODUCTION}

Multi-agent systems (MASs) have gained considerable attentions due to their extensive applications in many fields such as biology, physics, robotics, power grid, and so on. A prime concern for MASs is consensus, which means to attain an agreement regarding the state of all agents [1]. In most study on MASs [2]-[7], the structure of such systems was represented by the use of the Laplacian matrix, which is consisted of the adjacency and degree matrices. Here, there is room for further improvements for system structure in consensus analysis of MASs. For the details, because the foresaid matrices are corresponded with degree calculated by the number of nodes (agents in MASs) adjacent to it, the local information of network was utilized in the existing works. To compensate for this limitation, there is a need of study novel weighting approach to consensus protocol.

In this regard, in network theory, Newman [8] addresses question "Which are the most important or central nodes in network?" and introduces many answers to the question. Notably, it is remarkable that there are various concepts of centrality for the node, which were introduced first in the field of social science. In this paper, the betweenness and eigenvector centralities will be applied to novel frameworks for the consensus protocol for the first time to overcome the disadvantage of the Laplacian matrix. On a separate note, the concepts of two such centralities can be explained as follows:

- Betweenness centrality: One way of looking at centrality is by considering how important agents are in connecting other agents. In view of this point, betweenness centrality for a agent (or edge) is to compute the number of shortest paths between other agents that pass though the agent (or edge). For the details, the proposed

This research was supported by the Basic Science Research Program through the National Research Foundation of Korea (NRF) funded by the Ministry of Education, Science and Technology (2011-0009273). This work was also supported by the Human Resources Development of the Korea Institute of Energy Technology Evaluation and Planning (KETEP) grant funded by the Korea government Ministry of Trade, Industry \& Energy (20144030200450).

M.J. Park, and O.M. Kwon (Corresponding author) are with School of Electrical Engineering, Chungbuk National University, 1 Chungdae-ro, Cheongju 28644, Republic of Korea. Email:\{netgauss;madwind\}@ cbnu.ac.kr

A. Seuret is with CNRS, LAAS, 7 avenue du Colonel Roche, 31077 Toulouse, France. Email: aseuret@laas.fr consensus protocol with the betweenness centrality has the effect as the intermediary between each agent of edges, while the the existing protocol involves only the local information because the existing protocol corresponds with the degree determined by the number of agent adjacent to it. In other words, how central is an agent in connecting any pair of agents is considered in this work.

- Eigenvector centrality: In degree centrality, the node connected by the highest number of nodes is considered to be more important. However, in real networks, having more nodes does not by itself guarantee that someone is important. In other words, having more important nodes provides the stronger information. Thus, the eigenvector centrality is a natural extension of the simple degree centrality. In view of this respect, the agent which includes many important agents as central can be considered through the eigenvector centrality.

In addition to this, in view of centrality, the existing consensus protocol corresponds to the concept of degree centrality, which is called simple degree. With the concepts of the betweenness and eigenvector centralities, more structural information of network to analyze consensus problem for MASs can be weighted in the proposed consensus protocols. However, to the best of authors' knowledge, the utilization of these two centralities construct the consensus protocol has not been tackled in any other literature yet.

Motivated by the discussion above, this paper deals with the problem of a leader-following consensus problem in MASs with sampleddata by utilizing the betweenness and eigenvector centralities for the first time. Here, we consider MASs with fixed communication graph and synchronized sampling among the agents of the networks. At this time, the concept of sampled-data is considered as the communication constraint between each agent in network. In addition to this, because of the zero-order hold, continuous data are sampled before being used, a sampled-data appears discontinuous at sampling instants and continuous in other times [9]-[11]. To solve the problem mentioned above, by construction of a simple Lyapunov-Krasovskii functional and utilization of some mathematical techniques, the leader-following guaranteed cost consensus criterion with the existing protocol will be derived in Theorem 1. Based on the result of Theorem 1, two new consensus protocol design criteria which utilize the betweenness and eigenvector centralities will be proposed in Theorems 2 and 3 , respectively. Through one example, it will be shown that the consensus protocol design methods introduced in Theorems 2 and 3 can enhance the feasible region of consensus criteria by comparing maximum allowable interval bounds and guaranteed cost.

Notation: $\mathbb{R}, \mathbb{R}^{n}, \mathbb{R}^{m \times n}, \mathbb{S}^{n}$ and $\mathbb{S}_{+}^{n}$ denote, respectively, the sets of real numbers, $n$-vectors with the $l_{2}$-norm $\|\cdot\|, m \times n$ matrices, symmetric and positive definite $n \times n$ matrices. $I_{n}$ and 0 are $n \times n$ identity matrix and zero matrix of appropriate dimension. $X>0(<0)$ represents symmetric positive (negative) definite matrix. $X^{\perp}$ denotes a basis for the nullspace of $X$. $\operatorname{diag}\{\cdots\}$, $\operatorname{sym}\{X\}, \operatorname{col}\left\{x_{1}, \ldots, x_{n}\right\}$ and $\left\{y_{i}\right\}_{i=1}^{n}$ stand for, respectively, the (block) diagonal matrix, the sum of $X$ and $X^{T}$, the column vector with the vectors $x_{1}, \ldots, x_{2}$, and the set of the elements $y_{1}, \ldots, y_{n}$. The symmetric terms will be readily denoted by $\star$ when necessary. $X_{[f(t)]}$ means that its elements include the scalar value of $f(t)$ affinely.

\section{Preliminaries}

This section gives basic concepts and presents the problem formulation. 


\section{A. Graph theory}

The interaction topology of a network of agents is represented using a directed graph (digraph) $\mathcal{G}=(\mathcal{V}, \mathcal{E})$ with the set of nodes $\mathcal{V}=\{1,2, \ldots, N\}$ and the set of edges $\mathcal{E}=\{(i, j): i, j \in \mathcal{V}\} \subset$ $\mathcal{V} \times \mathcal{V}$. An adjacency matrix $\mathcal{A}=\left[a_{i j}\right]_{N \times N}$ of the digraph $\mathcal{G}$ is the matrix with nonnegative elements satisfying $a_{i i}=0$ and $a_{i j} \geq 0$. If there is an edge between $i$ and $j$, then the elements of matrix $\mathcal{A}$ described as $a_{i j}>0 \Leftrightarrow(i, j) \in \mathcal{E}$. The digraph $\mathcal{G}$ is said to be unweighted if all values of $a_{i j}$ for $(i, j) \in \mathcal{E}$ is 1 . A set of neighbors of agent $i$ is denoted by $\mathcal{N}_{i}=\{j \in \mathcal{V}:(i, j) \in \mathcal{E}\}$. A degree of node $i$ is denoted by $d_{o}(i)=\sum_{j \in \mathcal{N}_{i}} a_{i j}$. A degree matrix of digraph $\mathcal{G}$ is a diagonal matrix defined as $\mathcal{D}=\operatorname{diag}\left\{d_{o}(1), d_{o}(2), \ldots, d_{o}(N)\right\}$. The Laplacian matrix $\mathcal{L}$ of graph $\mathcal{G}$ is defined as $\mathcal{L}=\mathcal{D}-\mathcal{A}$. More details can be seen in [12].

The following lemma relates a nonnegative matrix with its directed graph, as well as supplying an algebraic characterization.

Lemma 1 (Perron-Frobenius theorem) [13]: Suppose that a directed graph $\mathcal{G}$ contains a spanning tree. $\mathcal{A}$ is irreducible and nonnegative, and $\rho(\mathcal{A})=\max \{|\lambda| ; \lambda$ is an eigenvalue of $\mathcal{A}\}>0$ is an algebraically simple eigenvalue of $\mathcal{A}$. Then, there is a positive vector $x$ such that $\mathcal{A} x=\rho(\mathcal{A}) x$.

\section{B. Problem formulation}

Consider the following model of second-order MASs:

$$
\begin{aligned}
& \dot{p}_{i}(t)=v_{i}(t), \\
& \dot{v}_{i}(t)=u_{i}(t), \quad i=1,2, \ldots, N,
\end{aligned}
$$

where $N$ is the number of agents, the subscript $i$ means the $i$ th agent, $p_{i}(t) \in \mathbb{R}, v_{i}(t) \in \mathbb{R}$ and $u_{i}(t) \in \mathbb{R}$ are the position, the velocity and the consensus protocol of agent $i$, respectively.

The leader for multi-agent system (1) is given by

$$
\dot{p}_{0}(t)=v_{0}(t), \dot{v}_{0}(t)=0 .
$$

Before proposing new consensus protocols, let us introduce the use of consensus protocol with sampled-data between each agent as

$$
\begin{aligned}
u_{i}(t)= & \sum_{j=1, j \neq i}^{N} a_{i j}\left[\left(p_{j}\left(t_{k}\right)-p_{i}\left(t_{k}\right)\right)+\left(v_{j}\left(t_{k}\right)-v_{i}\left(t_{k}\right)\right)\right] \\
& -b_{i}\left[\left(p_{i}(t)-p_{0}(t)\right)+\left(v_{i}(t)-v_{0}(t)\right)\right]
\end{aligned}
$$

for all $t \in\left[t_{k}, t_{k+1}\right)$, where $a_{i j}$ and $b_{i}$ are the interconnection weight defined as: $a_{i j}>0$ if agent $i$ is connected to agent $j$ and $a_{i j}=0$ otherwise, and where $b_{i}=1$ if leader is connected to agent $i$ and $b_{i}=0$ otherwise. Moreover, the information flow between each agent is assumed to be generated by a zero-order hold $(\mathrm{ZOH})$ function with a sequence of sampling instants $t_{k}$ satisfying $0=t_{0}<t_{1}<\cdots<$ $t_{k}<\cdots<\lim _{k \rightarrow \infty} t_{k}=+\infty$. When the sampling interval is constant, $t_{k+1}-t_{k}=h_{M}$, where $h_{M}$ is a known positive scalar. It should be noted that $\frac{d}{d t}\left(t-t_{k}\right)=1$ for $t \neq t_{k}$ and

$$
0 \leq t-t_{k} \leq h_{M}, \quad \forall t \in\left[t_{k}, t_{k+1}\right) .
$$

Remark 1: The consensus protocol (3) is based on the number of each agent's neighbors. For this reason, the protocol (3) corresponds to the concept of degree centrality in view of network analysis. Almost all research works for MASs use like the protocol (3) [1]-[7]. Hereinafter, the protocol (3) shall be referred as the existing protocol. To improve the consensus performance such as the transient consensus performance and the robustness on sampling interval in the sense of the maximum interval bound guaranteeing stability of system, from next section, two types of consensus protocol will be proposed by weighting the main role of each agent based on the betweenness and eigenvector centralities.
By the Laplacian matrix $\mathcal{L}=\left[l_{i j}\right]_{N \times N}$ associated with the structure of the information flow satisfying $l_{i j}=-a_{i j}$ for $i \neq j$ and $l_{i i}=-\sum_{j=1, j \neq i}^{N} l_{i j}$, system (1) is equivalent to

$$
\begin{aligned}
& \dot{p}_{i}(t)=v_{i}(t), \\
& \dot{v}_{i}(t)=-\sum_{j=1}^{N} l_{i j}\left[\bar{p}_{j}\left(t_{k}\right)+\bar{v}_{j}\left(t_{k}\right)\right]-b_{i}\left[\bar{p}_{i}(t)+\bar{v}_{i}(t)\right]
\end{aligned}
$$

for all $t \in\left[t_{k}, t_{k+1}\right)$, where $\bar{p}_{i}(t)=p_{i}(t)-p_{0}(t)$ and $\bar{v}_{i}(t)=v_{i}(t)-v_{0}(t)$.

Let us define $x(t)=\operatorname{col}\left\{\bar{p}_{1}(t), \ldots, \bar{p}_{N}(t), \bar{v}_{1}(t), \ldots, \bar{v}_{N}(t)\right\}$. Then, system (5) can be expressed in the matrix form

$$
\begin{aligned}
& \dot{x}(t)=\left(\mathbf{A}_{p}+\mathbf{A}_{u}\right) x(t)+\mathbf{B}_{\mathcal{L}} x\left(t_{k}\right), \quad \forall t \in\left[t_{k}, t_{k+1}\right), \\
& x(s)=x(0), \quad s \in\left[-h_{M}, 0\right],
\end{aligned}
$$

where $\mathcal{B}=\operatorname{diag}\left\{b_{1}, b_{2}, \ldots, b_{N}\right\}$,

$$
\mathbf{A}_{p}=\left[\begin{array}{ll}
0 & I_{N} \\
\hdashline 0 & 0
\end{array}\right], \mathbf{A}_{u}=\left[\begin{array}{c:c}
0 & 0 \\
-\mathcal{B} & -\mathcal{B}
\end{array}\right], \mathbf{B}_{\mathcal{L}}=\left[\begin{array}{cc}
0 & 0 \\
\hdashline \mathcal{L} & -\mathcal{L}
\end{array}\right] .
$$

The aim of this paper is to construct weighted consensus protocol for MASs (6). In other words, by the properties of the betweenness and eigenvector centralities, the proposed protocol $u_{i}(t)$ will be weighted to investigate the leader-following consensus problem for any initial condition if and only if $\lim _{t \rightarrow \infty}\left\|p_{i}(t)-p_{0}(t)\right\|=0$ and $\lim _{t \rightarrow \infty}\left\|v_{i}(t)-v_{0}(t)\right\|=0$ for $i=1,2, \ldots, N$. Moreover, to derive a main result, the following definition will be used.

Definition 1: Consider the multi-agent system (6). The consensus for system (6) is the guaranteed cost consensus if and only if there exists a Lyapunov-Krasovskii functional $V(t)$ such that the following condition holds

$$
V_{J}(t)=J(t)+\int_{0}^{\infty} \dot{V}(t) d t \leq 0
$$

where $J(t)>0$ is the quadratic cost function. It should be noted that if $V_{J}(t)<0$ from (7), then $\int_{0}^{\infty} \dot{V}(t) d t<0$. Moreover, from $J(t)+$ $\int_{0}^{\infty} \dot{V}(t) d t=J(t)+\left.V(t)\right|_{t \rightarrow \infty}-V(0) \leq 0$, if (6) is asymptotically stable, then since $\left.V(t)\right|_{t \rightarrow \infty}=0$, the $J(t)$ can be bounded as

$$
J(t) \leq V(0)
$$

In this work, the cost function $J(t)$ is constructed as follows:

$$
J(t)=\sum_{i=1}^{N} \int_{0}^{\infty}\left\{\gamma_{1}\left(\sum_{j=1}^{N} l_{i j}\left[\bar{p}_{j}(t)+\bar{v}_{j}(t)\right]\right)^{2}+\gamma_{2} u_{i}^{2}(t)\right\} d t
$$

for given positive scalars $\left\{\gamma_{i}\right\}_{i=1}^{2}$.

Remark 2: The consensus problem of the MASs with quadratic cost function (9) is similar to a typical LQR problem based on state errors between leader and each agent. Then, the quadratic cost function (9) can be regarded a leader-following consensus performances of agent $i$ and the energy consumption of the consensus protocol. Thus, leader-follower guaranteed cost consensus means that MASs achieve leader-follower consensus in company with a trade-off between the energy consumption of the consensus protocol and the leader-following consensus performances. In addition to this, the cost function (9) is immediately affected by $l_{i j}$. In point of view, through the novel consensus protocols to be proposed in next section, novel weights have more information about the interconnection between each agent than $l_{i j}$ of the existing 
protocol. For this reason, the guaranteed cost consensus condition will be influenced by the novel consensus protocols.

\section{MAIN RESULTS}

In this section, two types of novel consensus protocol are proposed by weighting the main role of each agent based on edge betweenness and eigenvector centralities.

\section{A. Leader-following guaranteed cost consensus}

In this subsection, the leader-following guaranteed cost consensus condition for system (6) is presented with the existing protocol (3) first. For simplicity of matrix and vector notations, some scalars and matrices are defined as follows:

$$
\begin{aligned}
& \zeta(t)=\operatorname{col}\left\{x(t), x\left(t_{k}\right), x\left(t-h_{M}\right), \dot{x}(t), \frac{1}{t-t_{k}} \int_{t_{k}}^{t} x(s) d s,\right. \\
& \left.\frac{1}{h_{M}-t+t_{k}} \int_{t-h_{M}}^{t_{k}} x(s) d s\right\} \\
& \Pi_{1\left[t-t_{k}\right]}=\left[e_{1},\left(t-t_{k}\right) e_{5}+\left(h_{M}-t+t_{k}\right) e_{6}\right], \Pi_{2}=\left[e_{4}, e_{1}-e_{3}\right] \text {, } \\
& \Pi_{3}=\left[e_{1}-e_{2}, e_{1}+e_{2}-2 e_{5}\right], \Pi_{4}=\left[e_{2}-e_{3}, e_{2}+e_{3}-2 e_{6}\right], \\
& \Theta=\left[\begin{array}{cc}
\operatorname{diag}\{R, 3 R\} & M \\
\hdashline & \operatorname{diag}\{R, 3 R\}
\end{array}\right], \\
& \Xi_{1\left[t-t_{k}\right]}=\operatorname{sym}\left\{\Pi_{1\left[t-t_{k}\right]} P \Pi_{2}^{T}\right\}+e_{1} Q e_{1}^{T}-e_{3} Q e_{3}^{T}+h_{M}^{2} e_{4} R e_{4}^{T} \text {, } \\
& \Xi_{2}=\left[\Pi_{3}, \Pi_{4}\right] \Theta\left[\Pi_{3}, \Pi_{4}\right]^{T} \text {, } \\
& \Omega_{1, \mathcal{L}}=\gamma_{1} e_{1}[\mathcal{L}, \mathcal{L}]^{T}[\mathcal{L}, \mathcal{L}] e_{1}^{T} \\
& \Omega_{2, \mathcal{L}}=\gamma_{2}\left[e_{1}, e_{2}\right]\left[\mathbf{A}_{u}, \mathbf{B}_{\mathcal{L}}\right]^{T}\left[\mathbf{A}_{u}, \mathbf{B}_{\mathcal{L}}\right]\left[e_{1}, e_{2}\right]^{T}, \\
& \Upsilon_{\mathcal{L}}=\left(\mathbf{A}_{p}+\mathbf{A}_{u}\right) e_{1}^{T}+\mathbf{B}_{\mathcal{L}} e_{2}^{T}-\left(I_{2} \otimes I_{N}\right) e_{4}^{T},
\end{aligned}
$$

where $\left\{e_{i}\right\}_{i=1}^{6} \in \mathbb{R}^{18 N \times 2 N}$ are the block entry matrices, e.g. $e_{2}^{T} \zeta(t)=x\left(t_{k}\right)$.

Now, the result is given by the following theorem:

Theorem 1: For given positive scalars $h_{M}$ and $\left\{\gamma_{i}\right\}_{i=1}^{2}$, all agents in the system (6) with the protocol (3) are consented to leader, if there exist matrices $P \in \mathbb{S}_{+}^{4 N}, Q \in \mathbb{S}_{+}^{2 N}, R \in \mathbb{S}_{+}^{2 N}$ and $M \in \mathbb{R}^{4 N \times 4 N}$ satisfying the following LMIs:

$$
\begin{aligned}
\Upsilon_{\mathcal{L}}^{\perp^{T}}\left(\hat{\boldsymbol{\Xi}}_{i}+\Omega_{\mathcal{L}}\right) \Upsilon_{\mathcal{L}}^{\perp} & <0, \quad i=1,2, \\
\Theta & >0
\end{aligned}
$$

where $\hat{\boldsymbol{\Xi}}_{\left[t-t_{k}\right]}=\Xi_{1\left[t-t_{k}\right]}-\Xi_{2}, \Omega_{\mathcal{L}}=\Omega_{1, \mathcal{L}}+\Omega_{2, \mathcal{L}}$ and $\left\{\hat{\boldsymbol{\Xi}}_{i}\right\}_{i=1}^{2}$ means the two vertices of $\hat{\boldsymbol{\Xi}}_{\left[t-t_{k}\right]}$ with the bounds of $0 \leq t-t_{k} \leq$ $h_{M}$; i.e., $\hat{\boldsymbol{\Xi}}_{1}=\hat{\boldsymbol{\Xi}}_{\left[t-t_{k}=0\right]}$ and $\hat{\boldsymbol{\Xi}}_{2}=\hat{\boldsymbol{\Xi}}_{\left[t-t_{k}=h_{U}\right]}$.

Then, guaranteed cost can be bounded as

$$
\begin{aligned}
J(t) \leq V(0)= & {\left[\begin{array}{c}
x(0) \\
\int_{-h_{M}}^{0} x(s) d s
\end{array}\right]^{T} P\left[\begin{array}{c}
x(0) \\
\int_{-h_{M}}^{0} x(s) d s
\end{array}\right] } \\
& +\int_{-h_{M}}^{0} x^{T}(s) Q x(s) d s .
\end{aligned}
$$

Proof: Consider the Lyapunov-Krasovskii functional candidate given by

$$
\begin{aligned}
V(t)= & {\left[\begin{array}{c}
x(t) \\
\int_{t-h_{M}}^{t} x(s) d s
\end{array}\right]^{T} P\left[\begin{array}{c}
x(t) \\
\int_{t-h_{M}}^{t} x(s) d s
\end{array}\right] } \\
& +\int_{t-h_{M}}^{t} x^{T}(s) Q x(s) d s \\
& +h_{M} \int_{t-h_{M}}^{t} \int_{s}^{t} \dot{x}^{T}(u) R \dot{x}(u) d u d s .
\end{aligned}
$$

Time-differentiating $V(t)$ leads to

$$
\dot{V}(t)=\zeta^{T}(t) \Xi_{1\left[t-t_{k}\right]} \zeta(t)-h_{M} \int_{t-h_{M}}^{t} \dot{x}^{T}(u) R \dot{x}(u) d u d s .
$$

Using the well-known lemmas for Wirtinger-based inequality in [14], [15] and the reciprocal convexity in [16], if inequality (11) holds, then the integral term is bounded as for any matrix $M$,

$$
\begin{aligned}
& h_{M} \int_{t-h_{M}}^{t} \dot{x}^{T}(s) R \dot{x}(s) d s \\
= & h_{M} \int_{t_{k}}^{t} \dot{x}^{T}(s) R \dot{x}(s) d s+h_{M} \int_{t-h_{M}}^{t_{k}} \dot{x}^{T}(s) R \dot{x}(s) d s \\
\geq & \frac{1}{\alpha(t)} \zeta^{T}(t) \Pi_{3} \operatorname{diag}\{R, 3 R\} \Pi_{3}^{T} \zeta(t) \\
& +\frac{1}{1-\alpha(t)} \zeta^{T}(t) \Pi_{4} \operatorname{diag}\{R, 3 R\} \Pi_{4}^{T} \zeta(t) \\
\geq & \zeta^{T}(t) \Xi_{2} \zeta(t),
\end{aligned}
$$

where $\alpha(t)=\frac{t-t_{k}}{h_{M}}$. Hence, an upper bound of $\dot{V}(t)$ is obtained as follows:

$$
\dot{V}(t) \leq \zeta^{T}(t)\left(\Xi_{1\left[t-t_{k}\right]}-\Xi_{2}\right) \zeta(t) .
$$

Moreover, the cost function (9) can be rewritten by matrix form

$$
J(t)=\int_{0}^{\infty} \zeta^{T}(t)\left(\Omega_{1, \mathcal{L}}+\Omega_{2, \mathcal{L}}\right) \zeta(t) d t
$$

From Definition 1 with (15) and (16), the guaranteed cost consensus condition can be obtained by

$$
\zeta^{T}(t)\left(\hat{\boldsymbol{\Xi}}_{\left[t-t_{k}\right]}+\Omega_{\mathcal{L}}\right) \zeta(t)<0 \text { s.t. } \Upsilon_{\mathcal{L}} \zeta(t)=0 .
$$

where $\hat{\Xi}_{\left[t-t_{k}\right]}=\Xi_{1\left[t-t_{k}\right]}-\Xi_{2}$ and $\Omega_{\mathcal{L}}=\Omega_{1, \mathcal{L}}+\Omega_{2, \mathcal{L}}$.

In succession, for $0 \leq t-t_{k} \leq h_{M}$, since the matrix $\Xi_{1\left[t-t_{k}\right]}$ is affinely dependent on $t-t_{k}$, the following relationship holds

$$
\hat{\boldsymbol{\Xi}}_{\left[t-t_{k}\right]}+\Omega_{\mathcal{L}}=(1-\alpha(t))\left(\hat{\boldsymbol{\Xi}}_{[0]}+\Omega_{\mathcal{L}}\right)+\alpha(t)\left(\hat{\boldsymbol{\Xi}}_{\left[h_{M}\right]}+\Omega_{\mathcal{L}}\right) .
$$

Since $\alpha(t)$ belongs to the interval $[0,1]$, verifying the previous condition is equivalent to

$$
\begin{aligned}
\hat{\boldsymbol{\Xi}}_{[0]}+\Omega_{\mathcal{L}}<0 & \text { s.t. } \Upsilon_{\mathcal{L}} \zeta(t)=0, \\
\hat{\boldsymbol{\Xi}}_{\left[h_{M}\right]}+\Omega_{\mathcal{L}}<0 & \text { s.t. } \Upsilon_{\mathcal{L}} \zeta(t)=0 .
\end{aligned}
$$

Finally, by the famous lemma of Finsler i) and ii) in [17], if the LMI (10) hold then the condition (17) is satisfied, which means that system (6) is asymptotically the leader-following guaranteed cost consensual. At this time, due to $x(s)=x(0), s \in\left[-h_{M}, 0\right]$ and the value of $x(0)$ is constant, the guaranteed cost can be bounded by (12). This completes our proof.

Remark 3: Consider the system (6) with cost function (9). For given sampling interval $h_{M}$, if the following optimization problem:

$$
\begin{aligned}
\min _{P, Q, R, M} J^{*} \quad \text { s.t. } & \text { LMIs (10), (11) and } \\
& \Psi\left(h_{M}\right)-J^{*} I_{2 N}<0,
\end{aligned}
$$

where

$$
\Psi\left(h_{M}\right)=\left[\begin{array}{c}
I_{2 N} \\
h_{M} I_{2 N}
\end{array}\right]^{T} P\left[\begin{array}{c}
I_{2 N} \\
h_{M} I_{2 N}
\end{array}\right]+h_{M} Q
$$

has solutions with $P, Q, R, M$ and $J^{*}$, then, the protocol (3) is the guaranteed cost consensus protocol for the system (6) and minimizes the upper bound of the cost function (9) as $J^{*}\|x(0)\|^{2}$, for any initial conditions $x(0)$. Moreover, from (12), it can be proved by holding the following inequality:

$$
\begin{aligned}
J(t) \leq V(0) & =\left[\begin{array}{c}
x(0) \\
h_{M} x(0)
\end{array}\right]^{T} P\left[\begin{array}{c}
x(0) \\
h_{M} x(0)
\end{array}\right]+h_{M} x^{T}(0) Q x(0) \\
& =x^{T}(0) \Psi\left(h_{M}\right) x(0)
\end{aligned}
$$


The following inequality:

$$
x^{T}(0) \Psi\left(h_{M}\right) x(0)<J^{*} x^{T}(0) x(0),
$$

which ensures $J(t)<J^{*}\|x(0)\|^{2}$, is equivalent to the LMI (18). Therefore, the minimization of $J^{*}$ means the minimization of the guaranteed cost function (9).

\section{B. Consensus protocol based on edge betweenness centrality}

In this subsection, a leader-following guaranteed cost consensus condition for system (6) is presented with the consensus protocol with the edge betweenness centrality.

Consider the following first novel consensus protocol constructed as follows

$$
\begin{aligned}
u_{i}(t)= & \sum_{\substack{j=1, j \neq i\\
}}^{N}\left(\frac{\bar{c}_{b, i j}}{\sum_{k=1, k \neq i}^{N} \bar{c}_{b, i k}}\right) a_{i j}\left[p_{j}\left(t_{k}\right)-p_{i}\left(t_{k}\right)\right. \\
& \left.+v_{j}\left(t_{k}\right)-v_{i}\left(t_{k}\right)\right] \\
& -b_{i}\left[\left(p_{i}(t)-p_{0}(t)\right)+v_{i}(t)-v_{0}(t)\right]
\end{aligned}
$$

where, $\bar{c}_{b, i j}$ is the edge betweenness centrality between agents $i$ and $j$ and its value is measured by

$$
\bar{c}_{b, i j}:=\sum_{k=1}^{N} \sum_{l=1, l \neq k}^{N} \frac{\mathrm{g}_{k l}\left(\mathrm{p}_{i j}\right)}{\mathrm{s}_{k l}} .
$$

Here, $\mathrm{p}_{i j}$ denotes the path from node $i$ to node $j, \mathbf{s}_{k l}$ is the number of shortest paths from node $k$ to $l$ (also known as information pathways) in the graph, and $\mathrm{g}_{k l}\left(\mathrm{p}_{i j}\right)$ is the number of these shortest paths which passes $\mathrm{p}_{i j}$.

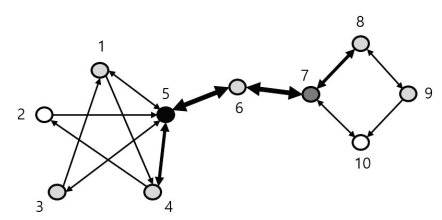

Fig. 1. Structure example for information flow.

Remark 4: From Figure 1, the thickness of edge is proportional to the edge betweenness centrality, while the node colors indicate the degrees (white means smallest degree). Thus, node 5 has the largest degree, whereas node 6 has the edge with the largest value of edge betweenness centrality. In this sense, the existing protocol (3) with degree utilized in many works [1]-[7] is only taking local information because the degree of node is determined by the number of nodes adjacent to it. Therefore, protocol (20) with edge betweenness centrality can be weighted by not only the local information but also the effect as the intermediary between each agent of edges.

More in detail, the edge betweenness centrality can be calculated as in the following example:

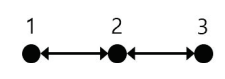

Fig. 2. 3-Agents Network.

- Computation of edge betweenness centrality: Consider 3-agents network drawn in Figure 2. For the edge $\mathrm{p}_{23}$, its betweenness centrality $\bar{c}_{b, 23}$ can be calculated by

$$
\begin{aligned}
\bar{c}_{b, 23}= & \sum_{k=1}^{3} \sum_{l=1, l \neq k}^{3} \frac{\mathrm{g}_{k l}\left(\mathrm{p}_{23}\right)}{\mathrm{s}_{k l}} \\
= & \frac{\mathrm{g}_{12}\left(\mathrm{p}_{23}\right)}{\mathrm{s}_{12}}+\frac{\mathrm{g}_{13}\left(\mathrm{p}_{23}\right)}{\mathrm{s}_{13}}+\frac{\mathrm{g}_{21}\left(\mathrm{p}_{23}\right)}{\mathrm{s}_{21}} \\
& +\frac{\mathrm{g}_{23}\left(\mathrm{p}_{23}\right)}{\mathbf{s}_{23}}+\frac{\mathrm{g}_{31}\left(\mathrm{p}_{23}\right)}{\mathbf{s}_{31}}+\frac{\mathrm{g}_{32}\left(\mathrm{p}_{23}\right)}{\mathbf{s}_{32}} \\
= & \frac{0}{1}+\frac{1}{1}+\frac{0}{1}+\frac{1}{1}+\frac{0}{1}+\frac{0}{1}=2 .
\end{aligned}
$$

It should be noted that, since the edge $\mathrm{p}_{23}$ has the direction from node 2 to node 3 , the terms $\mathrm{g}_{31}\left(\mathrm{p}_{23}\right)$ and $\mathrm{g}_{32}\left(\mathrm{p}_{23}\right)$ are zero.

Based on Theorem 1 and the novel consensus protocol (20), the following theorem is stated.

Theorem 2: For given positive scalars $h_{M}$ and $\left\{\gamma_{i}\right\}_{i=1}^{2}$, all agents in the system (6) with the protocol (20) follow the leader, if there exist matrices employed in Theorem 1 satisfying the LMIs (11) and

$$
\Upsilon_{\mathcal{L}_{b}}^{\perp}{ }^{T}\left(\hat{\boldsymbol{\Xi}}_{i}+\Omega_{\mathcal{L}_{b}}\right) \Upsilon_{\mathcal{L}_{b}}^{\perp}<0, \quad i=1,2,
$$

where $\mathcal{L}_{b}=\left[l_{b, i j}\right]_{N \times N}$ is the Laplacian matrix weighted with edge betweenness centrality and associated with the structure of the information flow satisfying $l_{b, i j}=-\left(\frac{\bar{c}_{b, i j}}{\sum_{k=1, k \neq i}^{N} \bar{c}_{b, i k}}\right) a_{i j}$ for $i \neq j$ and $l_{b, i i}=-\sum_{j=1, j \neq i}^{N} l_{b, i j}$.

Proof: By replacing $\mathcal{L}$ in Theorem 1 with $\mathcal{L}_{b}$, LMIs (21) can be easily obtained.

\section{Consensus protocol based on eigenvector centrality}

In this subsection, a leader-following guaranteed cost consensus condition for system (6) is presented with the consensus protocol with the eigenvector centrality.

Consider a second novel consensus protocol constructed by

$$
\begin{aligned}
u_{i}(t)= & \sum_{j=1, j \neq i}^{N} c_{e, j} a_{i j}\left[p_{j}\left(t_{k}\right)-p_{i}\left(t_{k}\right)+v_{j}\left(t_{k}\right)-v_{i}\left(t_{k}\right)\right] \\
& -b_{i}\left[\left(p_{i}(t)-p_{0}(t)\right)+v_{i}(t)-v_{0}(t)\right]
\end{aligned}
$$

where $c_{e, j}$ is called as the eigenvector centrality of agent $j$, and its value can be obtained from the eigenvector defined as $\mathbf{C}_{e}=\operatorname{col}\left\{c_{e, 1}, c_{e, 2}, \ldots, c_{e, N}\right\}$ of the adjacency matrix $\mathcal{A}$ of the graph $\mathcal{G}$ corresponding to the largest eigenvalue of $\mathcal{A}$.

Remark 5: The eigenvector centrality is proportional to the sum of the centralities of the adjacent agents. In other words, the eigenvector centrality generalizes degree by incorporating the importance of the adjacent agents. Moreover, to weight the eigenvector centrality, the graph $\mathcal{G}$ should be strongly connected. By the use of Lemma 1 , we can compute the eigenvalues of $\mathcal{A}$ and then select the largest eigenvalue. Then, the corresponding eigenvector is $\mathbf{C}_{e}$. Thus, by Lemma 1 , all the components of $\mathbf{C}_{e}$ will be positive, and the elements of this vector correspond to the values of eigenvector centrality for the graph $\mathcal{G}$. As as result, in the proposed protocol (22), the weight of each edge will be the value of the eigenvector centrality of the tail agent.

More in detail, eigenvector centrality can be calculated like the following example:

- Computation of eigenvector centrality: Consider 3-agent network drawn in Figure 2. The adjacency matrix is $\mathcal{A}=\left[\begin{array}{lll}0 & 1 & 0 \\ 1 & 0 & 1 \\ 0 & 1 & 0\end{array}\right]$ and its eigenvalues are $-\sqrt{2}, 0$ and $\sqrt{2}$. For eigenvector centrality, with Proposition 1, the largest eigenvalue is selected as 
TABLE I

LAPLACIAN MATRICES CORRESPOND TO EACH PROTOCOL.

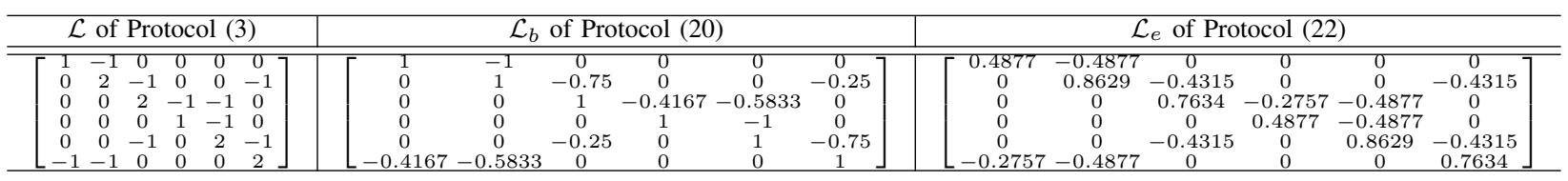

$\sqrt{2}$. The corresponding eigenvector is the eigenvector centrality vector and is

$$
\mathbf{C}_{e}=\operatorname{col}\left\{c_{e, 1}, c_{e, 2}, c_{e, 3}\right\}=\operatorname{col}\{1 / 2, \sqrt{2} / 2,1 / 2\} .
$$

Now, from the result of Theorem 1 and a novel consensus protocol (22) which utilizes eigenvector centrality, the following theorem is introduced.

Theorem 3: For given positive scalars $h_{M}$ and $\left\{\gamma_{i}\right\}_{i=1}^{2}$, all agents in the system (6) with the protocol (22) follow the leader, if there exist matrices employed in Theorem 1 satisfying the LMIs (11) and

$$
\Upsilon_{\mathcal{L}_{e}}^{\perp}{ }^{T}\left(\hat{\boldsymbol{\Xi}}_{i}+\Omega_{\mathcal{L}_{e}}\right) \Upsilon_{\mathcal{L}_{e}}^{\perp}<0, \quad i=1,2,
$$

where $\mathcal{L}_{e}=\left[l_{e, i j}\right]_{N \times N}$ is the Laplacian matrix weighted with the eigenvector centrality and associated with the structure of the information flow satisfying $l_{e, i j}=-c_{e, j} a_{i j}$ for $i \neq j$ and $l_{e, i i}=$ $-\sum_{j=1, j \neq i}^{N} l_{e, i j}$.

Proof: By replacing $\mathcal{L}$ in Theorem 1 with $\mathcal{L}_{e}$, it can be easy derived conditions (23).

\section{ILLUSTRATIVE EXAMPLE}

In this section, an illustrative example is introduced to show the improvements of the proposed protocols.

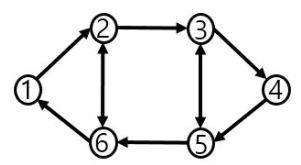

Fig. 3. 6-Agents Network (Source: Taken from [2]).

Consider the information flow consisting of a 6-agent network drawn by Figure 3, and the corresponding Laplacian matrix is presented in Table I. For comparison, the values of $\left\{\gamma_{i}\right\}_{i=1}^{2}$ are fixed as 1 , and to nullify the influence of selecting the interconnection between the leader and any agent; in other words, to confirm only the effect of the proposed protocols (20) and (22), it is assumed that all agents are connected to the leader, i.e., $\mathcal{B}=\operatorname{diag}\{1,1,1,1,1,1\}$.

From the following three points of view, the differences between the existing protocol (3) and the proposed protocols (20) and (22) are analyzed as follows:

- Maximum sampling interval: From Table II, the maximum sampling interval bounds considered by the proposed consensus protocols (20) and (22) are larger than the one with the existing protocol (3). This means that through the proposed protocols introduced in Theorems 2 and 3, the maximum sampling intervals guaranteed the stability of MASs can be increased comparing with the protocol (3). In order to confirm the results of Table II, the simulation result is drawn in Figure 4. For the details, from this figure, when the sampling interval $h_{M}=0.70$, the initial condition of the leader $p_{0}(0)=0$ with its velocity $v_{0}(t)=1$ and the initial positions and velocities of agents $p_{i}(0)=i$ and $v_{i}(0)=0$, where $i=1,2, \ldots, 6$, the proposed two protocols ensure the consensus of MASs, whereas the use of protocol does not.
TABLE II

MAXIMUM INTERVAL BOUNDS WITH FIXED $\gamma_{1}=\gamma_{2}=1$.

\begin{tabular}{c|ccc}
\hline $\begin{array}{c}\text { Methods } \\
(\text { Protocols })\end{array}$ & $\begin{array}{c}\text { Theorem 1 } \\
((3) \text { with } \mathcal{L})\end{array}$ & $\begin{array}{c}\text { Theorem 2 } \\
\left((20) \text { with } \mathcal{L}_{b}\right)\end{array}$ & $\begin{array}{c}\text { Theorem 3 } \\
\left((22) \text { with } \mathcal{L}_{e}\right)\end{array}$ \\
\hline \hline$h_{M}$ & 0.43 & 0.70 & 0.87
\end{tabular}

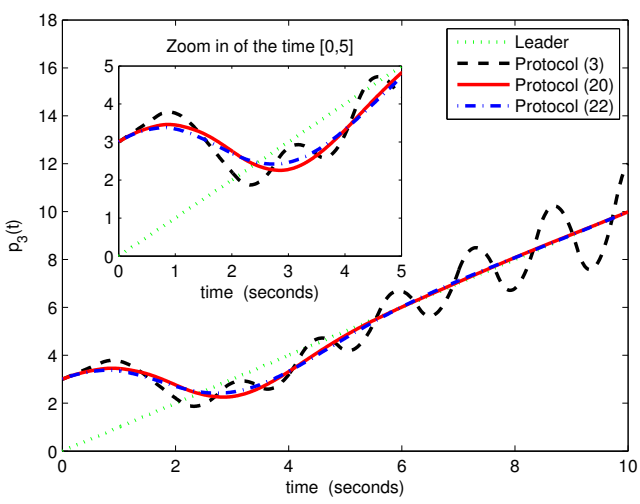

Fig. 4. Trajectories of agent 3 with each protocol when $h_{M}=0.70$.
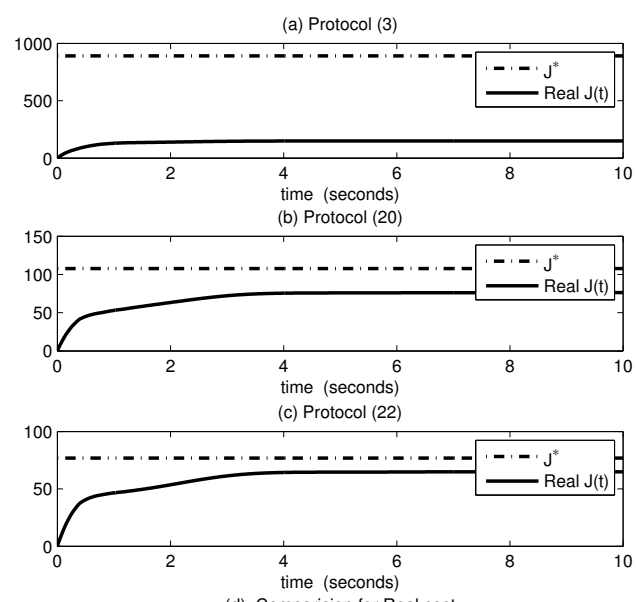

(d) Comparision for Real cost

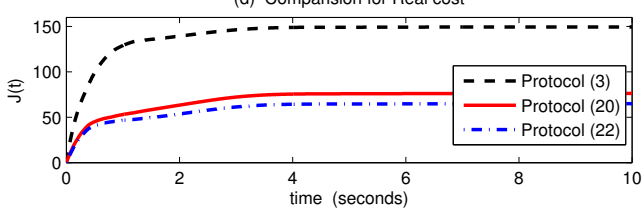

Fig. 5. Trajectories of the related cost with each protocol when $h_{M}=0.4$. 
TABLE III

OPTIMAL COSTS WITH FIXED $\gamma_{1}=\gamma_{2}=1, h_{M}=0.4$.

\begin{tabular}{c|ccc}
\hline Methods & Theorem 1 & Theorem 2 & Theorem 3 \\
\hline \hline$J^{*}$ & 891.30 & 107.69 & 76.86
\end{tabular}

- Guaranteed cost: With Remark 3, the optimal guaranteed costs with the fixed $h_{M}=0.4$ are listed in Table III and the real costs are drawn in Figure 5. The initial conditions used at this time are mentioned in Maximum sampling interval. The existing protocol (3) is the leftmost of list of larger-to-smaller guaranteed costs given by Table III. This means that the use of the existing protocol (3) is required the largest value of the guaranteed cost. Moreover, from the first result on the maximum sampling interval, the cost with the protocol (22) proposed with the eigenvector centrality is smaller than the ones with other protocols. Thus, in the guaranteed cost of view, the proposed protocol (22) is also more effective than others.

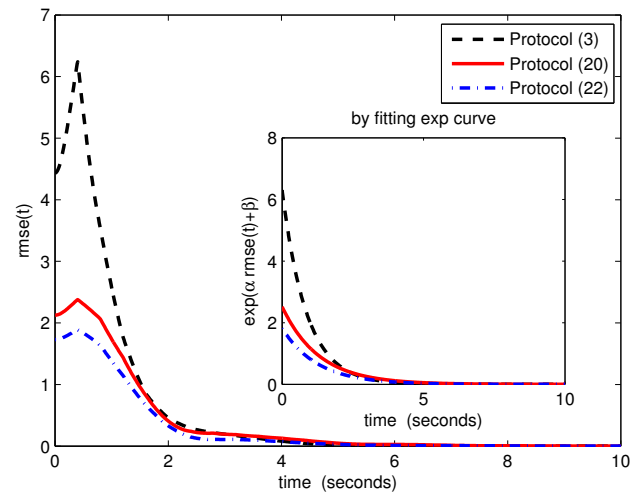

Fig. 6. Trajectories of root mean square of error of the positions and the velocities $\mathrm{rmse}(t)$ with each protocol when $h_{M}=0.4$.

- Transient consensus performance: From Figure 6, based on the root mean square of error of the positions and the velocities between the leader and each agent defined as in an equation $\operatorname{rmse}(t)=\sqrt{\frac{\sum_{i=1}^{5}\left\{\left(\sum_{j=1}^{5} l_{i j} \bar{p}_{j}(t)\right)^{2}+\left(\sum_{j=1}^{N} l_{i j} \bar{v}_{j}(t)\right)^{2}\right\}}{5}}$, it can be known that there is a difference in the transient consensus performance with three protocols. At this time, the function rmse $(t)$ is considered with $l_{i j}$ like as the cost function (9). In addition to this, to the clarity of the consensus tendency, by the curve fitting method, the exponential curves of the form $e^{\alpha \cdot \operatorname{rmse}(t)+\beta}$, where $\alpha$ and $\beta$ are scalars, are redrawn in Figure 6.

To sum up, by and large, the most effective weighted framework is the protocol (22) proposed with centrality centrality. Moreover, under the tolerance on the narrow difference between the proposed protocols (20) and (22), the protocol (20) proposed with the edge betweenness centrality can also be valuable to robustness with respect to aperiodic sampling and to guaranteed cost optimization.

\section{Conclusions}

In this paper, the leader-following guaranteed cost consensus problem for second-order MASs under sampling interval has been investigated. To improve the consensus performance such as the robustness on sampling interval, the guaranteed cost and the transient consensus performance, the weighted consensus protocols were proposed by calculating the betweenness and eigenvector centralities for agent and edge in network. To achieve this, by constructing the simple Lyapunov-Krasovskii functional, the sufficient conditions for such problem with the proposed protocols have been derived in terms of LMIs. One numerical example has been given to show the effectiveness of the proposed protocols. Moreover, for the connection between the leader and the selected agent, our future works will focus on grafting the pinning control onto this work.

\section{REFERENCES}

[1] R. Olfati-Saber, J.A. Fax, R.M. Murray, "Consensus and cooperation in networked multi-agent systems," Proceedings of The IEEE, vol. 95, pp.215-233, 2007.

[2] R. Olfati-Saber, R.N. Murray, "Consensus problems in networks of agents with switching topology and time-delays," IEEE Trans. Autom. Control, vol.49, pp.1520-1533, 2004.

[3] W. Ren, "On consensus algorithms for double-integrator dynamics," IEEE Trans. Autom. Control, vol.58, pp.1503-1509, 2008.

[4] H. Kim, H. Shim, J.H. Seo, "Output consensus of heterogeneous uncertain linear multi-agent systems," IEEE Trans. Autom. Control, vol.56, pp.200-206, 2011.

[5] W. Yu, G. Ghen, M. Gao, "Some necessary and sufficient conditions for second-order consensus in multi-agent dynamical systems," Automatica, vol.46, pp.1089-1095, 2010.

[6] K.K. Oh, M.-C. Park, H.-S. Ahn, "A survey of multi-agent formation control," Automatica, vol.53, pp.424-440, 2015.

[7] M.J. Park, O.M. Kwon, Ju H. Park, S.M. Lee, E.J. Cha, "Randomly changing leader-following consensus control for Markovian switching multi-agent systems with interval time-varying delays," Nonlinear Anal.Hybrid Syst., vol.12, pp.117-131, 2014.

[8] M.E.J. Newman, Networks: An Introduction. Oxford, UK: Oxford University Press, 2010.

[9] G.F. Franklin, J.D. Powell, M. Workman, Digital Control of Dynamic Systems. New York: Addison-Wesley, 1998.

[10] A. Seuret, M.M. Peet, "Stability analysis of sampled-data systems using sum of squares," IEEE Trans. Autom. Control, vol.58, pp.1620-1625, 2013.

[11] E. Fridman, "A refined input delay approach to sampled-data control", Automatica, vol.46, pp.421-427, 2010.

[12] C. Godsil and G. Royle, Algebraic Graph Theory. New York: SpringerVerlag, 2001.

[13] R.A. Horn and C.R. Johnson, Matrix Analysis. New York: Cambride University Press, 1990.

[14] A. Seuret, F. Gouaisbaut, "Wirtinger-based integral inequality: application to time-delay systems," Automatica, vol.49, pp.2860-2866, 2013.

[15] M.J. Park, O.M. Kwon, J.H. Park, S.M. Lee, E.J. Cha, "Stability of time-delay systems via Wirtinger-based double integral inequality," Automatica, vol.55, pp.204-208, 2015.

[16] P. Park, J.W. Ko, C.K. Jeong, "Reciprocally convex approach to stability of systems with time-varying delays," Automatica, vol.47, pp.235-238, 2011.

[17] M.C. de Oliveira, R.E. Skelton, Stability tests for constrained linear systems. Berlin: Springer-Verlag, 2001.

[18] S. Boyd, L.E. Ghaoui, E. Feron, V. Balakrishnan, Linear Matrix Inequalities in System and Control Theory Philadelphia: SIAM, 1994. 